\title{
On the multiplicative independence of binomial coefficients
}

\author{
by \\ Jianguo XiA and Hourong Qin (Nanjing)
}

1. Introduction. Let $F$ be a finite extension of the field $\mathbb{Q}$ of rational numbers with the ring of integers $O_{F}$. For a finite set $S$ of primes of $F$ containing all infinite primes, we use $U_{S}$ to denote the group of $S$-units of $F$, i.e., $a \in U_{S}$ if and only if $\operatorname{ord}_{p}(a)=0$ for all primes $p$ of $F$ not belonging to $S$. We call the elements in the set $W_{S}:=U_{S} \cap\left(1-U_{S}\right)$ good $S$-units. It is known that $W_{S}$ is finite (see [2, Theorem 1]).

Let $S=\{\infty, 2,3, \ldots, p\}$ be the set of the first $n$ prime numbers together with $\infty$, i.e., $p=p_{n}$. For $1 \leq k \leq p / 2$, put $q_{k}=k /(p-k)$. It is clear that every $q_{k}$ is a good $S$-unit.

Two open problems were raised by Browkin in [1].

(a) Is it true that exactly $n-1$ numbers among $q_{k}$ are multiplicatively independent?

(b) Is the index $\left(U_{S} \wedge U_{S}: \lambda\left(A\left(W_{S}\right)\right)\right)$ finite? Equivalently, are the free ranks of both groups equal?

We remark that a positive answer to problem (a) in fact answers problem (b) affirmatively. Browkin claimed that the answer to problem (a) is positive when $p \leq 47$ or $p=101$.

Let $G$ be the subgroup of $\mathbb{Q}^{*}$ generated by the binomial coefficients $\left(\begin{array}{c}p-1 \\ i\end{array}\right), i=1, \ldots,[p / 2]$. Because $q_{k}=\left(\begin{array}{c}p-1 \\ k-1\end{array}\right) /\left(\begin{array}{c}p-1 \\ k\end{array}\right)$ and $\left(\begin{array}{c}p-1 \\ k\end{array}\right)=\left(q_{1} \cdots q_{k}\right)^{-1}$, $G$ is equal to the subgroup of $\mathbb{Q}^{*}$ generated by good $S$-units $q_{k}, k=1, \ldots$, $[p / 2]$. We see that exactly $n-1$ numbers among $q_{k}$ are multiplicatively independent if and only if the rank of $G$ is $n-1$.

In this paper, we prove the following theorem, which means that the answers to the two problems mentioned above are positive.

2000 Mathematics Subject Classification: 05A10, 11B65, 11R27.

Key words and phrases: rank of subgroups, good $S$-units.

This work was supported by the National Natural Science Foundation of China 10471118, SRFDP, the Jiangsu Natural Science Foundation Bk2002023, the National Distinguished Youth Science Foundation of China Grant and the 973 Grant. 
THEOREM. Let $p=p_{n}$ be the $n$th prime and $G$ the subgroup of $\mathbb{Q}^{*}$ generated by the binomial coefficients $\left(\begin{array}{c}p-1 \\ i\end{array}\right), i=1, \ldots,[p / 2]$. Then the rank of $G$ is $n-1$.

2. Proof of Theorem. It is evident that the rank of $G$ does not exceed $n-1$ since every binomial coefficient $\left(\begin{array}{c}p-1 \\ i\end{array}\right)$ has the form

$$
\left(\begin{array}{c}
p-1 \\
i
\end{array}\right)=p_{1}^{m_{1}} \cdots p_{n-1}^{m_{n-1}}
$$

for some integers $m_{1}, \ldots, m_{n-1} \in \mathbb{Z}$.

In order to prove that the rank of $G$ is exactly $n-1$, we only need to prove the following assertion:

There exist integers $e_{k 1}, \ldots, e_{k k}$ with $e_{k k} \neq 0$ such that $2^{e_{k 1}} 3^{e_{k 2}} \cdots p_{k}^{e_{k k}} \in$ $G$ for $1 \leq k \leq n-1$.

The case $n=1$, i.e., $p_{n}=2$, is trivial.

Now suppose that $p=p_{n}$ is an odd prime. First let us prove that the assertion is true for $k=1$, i.e., $2^{e_{11}} \in G$ for some $e_{11} \in \mathbb{Z}$ with $e_{11} \neq 0$.

Set $b_{0}=1, a_{1}=b_{0} \cdot 2^{m_{1}}$, where $m_{1} \in \mathbb{Z}$ and $2^{m_{1}}$ is the highest power of 2 less than $p$. Then $p / 2<a_{1}<p$. Set $b_{1}=p-a_{1}$. Then $0<b_{1}<p / 2$. Set $a_{2}=b_{1} \cdot 2^{m_{2}}$, where $m_{2} \in \mathbb{Z}$ and $2^{m_{2}}$ is the highest power of 2 less than $p / b_{1}$. Then $p / 2<a_{2}<p$. In general, we define $a_{i}=b_{i-1} \cdot 2^{m_{i}}, b_{i}=p-a_{i}$ by induction on $i$, where $m_{i} \in \mathbb{Z}$ and $2^{m_{i}}$ is the highest power of 2 less than $p / b_{i-1}$. Then $p / 2<a_{i}<p$. Thus $b_{i}<p / 2$ and $m_{i}>0$ for any $i$.

Notice that each of $a_{i}$ is a positive integer less than $p$, so there exist $i$ and $j$ with $i<j$ such that $a_{i}=a_{j}$. Thus

$$
\begin{aligned}
\frac{a_{i}}{p-a_{i}} \cdot \frac{a_{i+1}}{p-a_{i+1}} \cdots \frac{a_{j-1}}{p-a_{j-1}} & =\frac{a_{i}}{b_{i}} \cdot \frac{a_{i+1}}{b_{i+1}} \cdots \frac{a_{j-1}}{b_{j-1}} \\
& =\frac{a_{i+1}}{b_{i}} \cdot \frac{a_{i+2}}{b_{i+1}} \cdots \frac{a_{j}}{b_{j-1}}=2^{m_{i+1}+\cdots+m_{j}} .
\end{aligned}
$$

Set $e_{11}=m_{i+1}+\cdots+m_{j}$. Then $e_{11}>0$ and $2^{e_{11}} \in G$. So the assertion is true for $k=1$.

Next let us prove that the assertion is true for $k=2$, i.e., $2^{e_{21}} 3^{e_{22}} \in G$ for some $e_{21}, e_{22} \in \mathbb{Z}$ with $e_{22} \neq 0$.

Set $b_{0}=1, a_{1}=b_{0} \cdot 3^{m_{1}}$, where $m_{1} \in \mathbb{Z}$ and $3^{m_{1}}$ is the highest power of 3 less than $p$. Then $p / 3<a_{1}<p$. Let $p-a_{1}=2^{n_{1}} b_{1}$ with $b_{1}$ odd. Since $a_{1}$ is odd, $n_{1} \geq 1$. So $b_{1} \leq\left(p-a_{1}\right) / 2<p / 3$. Set $a_{2}=b_{1} \cdot 3^{m_{2}}$, where $m_{2} \in \mathbb{Z}$ and $3^{m_{2}}$ is the highest power of 3 less than $p / b_{1}$. Then $p / 3<a_{2}<p$. Since $b_{1}<p / 3, m_{2} \geq 1$. Let $p-a_{2}=2^{n_{2}} b_{2}$ with $b_{2}$ odd. Then $n_{2} \geq 1$. In general, we define $a_{i}$ and $b_{i}$ by induction on $i: a_{i}=b_{i-1} \cdot 3^{m_{i}}$, where $m_{i} \in \mathbb{Z}$ and $3^{m_{i}}$ is the highest power of 3 less than $p / b_{i-1}$. Let $p-a_{i}=2^{n_{i}} b_{i}$ with $b_{i}$ odd. It is easy to prove by induction on $i$ that $b_{i}<p / 3$. So $m_{i}$ is a positive integer. 
Notice that each of $a_{i}$ is a positive integer less than $p$, so there exist $i$ and $j$ with $i<j$ such that $a_{i}=a_{j}$. Thus

$$
\frac{a_{i}}{b_{i}} \cdot \frac{a_{i+1}}{b_{i+1}} \cdots \frac{a_{j-1}}{b_{j-1}}=\frac{a_{i+1}}{b_{i}} \cdot \frac{a_{i+2}}{b_{i+1}} \cdots \frac{a_{j}}{b_{j-1}}=3^{m_{i+1}+\cdots+m_{j}} .
$$

So

$$
\frac{a_{i}}{p-a_{i}} \cdot \frac{a_{i+1}}{p-a_{i+1}} \cdots \frac{a_{j-1}}{p-a_{j-1}}=2^{-\left(n_{i}+\cdots+n_{j-1}\right)} \cdot 3^{m_{i+1}+\cdots+m_{j}} .
$$

Set $e_{21}=-\left(n_{i}+\cdots+n_{j-1}\right), e_{22}=m_{i+1}+\cdots+m_{j}$. Then $e_{22}>0$ and $2^{e_{21}} 3^{e_{22}} \in G$. So the assertion is true for $k=2$.

Finally, let us prove that the assertion is true for $3 \leq k \leq n-1$, i.e., there exist integers $e_{k 1}, \ldots, e_{k k}$ with $e_{k k} \neq 0$ such that $2^{e_{k 1}} 3^{e_{k 2}} \cdots p_{k}^{e_{k k}} \in G$ for $3 \leq k \leq n-1$.

Let $q=p_{k}$. Set $b_{0}=1, a_{1}=b_{0} q^{m_{1}}\left(2 l_{1}-1\right)$, where $m_{1} \in \mathbb{Z}$ and $q^{m_{1}}$ is the highest power of $q$ less than $p, l_{1}$ the largest integer with $b_{0} q^{m_{1}}\left(2 l_{1}-1\right)$ less than $p$. Then $p / q<b_{0} q^{m_{1}}<p$ and $b_{0} q^{m_{1}}\left(2 l_{1}-1\right)<p<b_{0} q^{m_{1}}\left(2 l_{1}+1\right)$. Let $p-a_{1}=2^{n_{1}} b_{1}$ with $b_{1}$ odd. Then $n_{1} \geq 1$. In general, we define $a_{i}$ and $b_{i}$ by induction on $i: a_{i}=b_{i-1} q^{m_{i}}\left(2 l_{i}-1\right)$ with $p / q<b_{i-1} q^{m_{i}}<p$ and $b_{i-1} q^{m_{i}}\left(2 l_{i}-1\right)<p<b_{i-1} q^{m_{i}}\left(2 l_{i}+1\right), p-a_{i}=2^{n_{i}} b_{i}$ with $b_{i}$ odd. Clearly $2 l_{i}-1<q$ for $i \geq 1$. Since $q$ is odd, $2 l_{i}+1 \leq q$ for $i \geq 1$.

Since each of $a_{i}$ is a positive integer less than $p$, there exist $i$ and $j$ with $i<j$ such that $a_{i}=a_{j}$. Thus

$$
\begin{aligned}
\frac{a_{i}}{b_{i}} \cdot \frac{a_{i+1}}{b_{i+1}} \cdots \frac{a_{j-1}}{b_{j-1}} & =\frac{a_{i+1}}{b_{i}} \cdot \frac{a_{i+2}}{b_{i+1}} \cdots \frac{a_{j}}{b_{j-1}} \\
& =q^{m_{i+1}+\cdots+m_{j}}\left(2 l_{i+1}-1\right) \cdots\left(2 l_{j}-1\right)
\end{aligned}
$$

and

$$
\begin{aligned}
\frac{a_{i}}{p-a_{i}} \cdot \frac{a_{i+1}}{p-a_{i+1}} & \cdots \frac{a_{j-1}}{p-a_{j-1}} \\
= & \frac{a_{i}}{b_{i}} \cdot \frac{a_{i+1}}{b_{i+1}} \cdots \frac{a_{j-1}}{b_{j-1}} 2^{-\left(n_{i}+\cdots+n_{j-1}\right)} \\
= & q^{m_{i+1}+\cdots+m_{j}}\left(2 l_{i+1}-1\right) \cdots\left(2 l_{j}-1\right) \cdot 2^{-\left(n_{i}+\cdots+n_{j-1}\right)} .
\end{aligned}
$$

We claim that $m_{i+1}+\cdots+m_{j}>0$. In fact, if $m_{i+1}+\cdots+m_{j}$ $=0$, then $m_{i+1}=\cdots=m_{j}=0$. Since $a_{i}=a_{j}$, we have $b_{i}=b_{j}$ and $a_{i+1}=a_{j+1}$, which means that $l_{j+1}=l_{i+1}$. Since $m_{i+1}=m_{i+2}=0$, $a_{i+1}=b_{i}\left(2 l_{i+1}-1\right), a_{i+2}=b_{i+1}\left(2 l_{i+2}-1\right)$. By definition of $l_{i+1}$ we have

$$
\frac{2 l_{i+1}-1}{2 l_{i+1}+1} p<a_{i+1}<p .
$$

Notice that $n_{i+1} \geq 1$, hence

$$
0<b_{i+1}=\frac{p-a_{i+1}}{2^{n_{i+1}}} \leq \frac{p-a_{i+1}}{2}<\frac{1}{2 l_{i+1}+1} p .
$$


So $2 l_{i+2}-1 \geq 2 l_{i+1}+1$, hence $l_{i+2}>l_{i+1}$. Continuing this process, we finally get $l_{j+1}>l_{j}>l_{j-1}>\cdots>l_{i+2}>l_{i+1}$, which is a contradiction to $l_{j+1}=$ $l_{i+1}$. On the other hand, $m_{i+1}+\cdots+m_{j} \geq 0$, hence $m_{i+1}+\cdots+m_{j}>0$.

Since $2 l_{i+1}-1<q, \ldots, 2 l_{j}-1<q,\left(2 l_{i+1}-1\right) \cdots\left(2 l_{j}-1\right)$ has the form $3^{e_{k 2}} \cdots p_{k-1}^{e_{k, k-1}}$ for some $e_{k 2}, \ldots, e_{k, k-1} \in \mathbb{Z}$. Let $e_{k 1}=-\left(n_{i}+\cdots+n_{j-1}\right)$, $e_{k k}=m_{i+1}+\cdots+m_{j}$. Then $2^{e_{k 1}} 3^{e_{k 2}} \cdots p_{k-1}^{e_{k, k-1}} p_{k}^{e_{k k}} \in G$ and $e_{k k}>0$. So the assertion is true for $3 \leq k \leq n-1$.

This completes the proof.

Acknowledgements. The authors are greatly indebted to the referee for careful reading of this paper and detailed suggestions for improvement.

\section{References}

[1] J. Browkin, K-theory, cyclotomic equations, and Clausen's function, in: Structural Properties of Polylogarithms, L. Lewin (ed.), Math. Surveys Monogr. 37, Amer. Math. Soc., Providence, RI, 1991, 233-273.

[2] J.-H. Evertse, On equations in S-units and the Thue-Mahler equation, Invent. Math. 75 (1984), 561-584.

Jianguo Xia

Department of Mathematics

Nanjing Normal University

Nanjing, 210097, China

E-mail: jgxia@pine.njnu.edu.cn
Hourong Qin Department of Mathematics Nanjing University Nanjing, 210093, China E-mail: hrqin@nju.edu.cn

Received on 26.4.2004

and in revised form on 23.9.2004 